City University of New York (CUNY)

CUNY Academic Works

\title{
THEORETICAL ANALYSIS OF THE LEAKAGE THROUGH THE CEMENT LINE OF A SINGLE OSTEON
}

Gaffar Gailani

CUNY New York City College of Technology

Stephen Cowin

cuNY City College

\section{How does access to this work benefit you? Let us know!}

More information about this work at: https://academicworks.cuny.edu/ny_pubs/570

Discover additional works at: https://academicworks.cuny.edu

This work is made publicly available by the City University of New York (CUNY).

Contact: AcademicWorks@cuny.edu 


\title{
THEORETICAL ANALYSIS OF THE LEAKAGE THROUGH THE CEMENT LINE OF A SINGLE OSTEON
}

\author{
GAFFAR GAILANI \\ Center of Medical Devices and Additive Manufacturing \\ Dept. of Mechanical Engineering \& Industrial Design Tech. \\ New York City College of Technology of the \\ City University of New York \\ 300 Jay St, V518 \\ Brooklyn, NY 11201 \\ USA \\ ggailani@citytech.cuny.edu \\ STEPHEN COWIN \\ Dept. of Mechanical and Biomedical Engineering \\ City College of New York of the \\ City University of New York \\ 160 Convent Ave \\ New York, NY 10031 \\ USA
}

\begin{abstract}
This work focuses on the Lacunar Canalicular Porosity (PLC) of cortical bone which includes the osteons. Osteons are semi cylindrical porous structures saturated with fluid within the bone and are approximately 250 micrometers in diameter. The outer boundary of the osteon is called the cement line. Some studies suggested that the cement line is less highly mineralized and produced evidence that it has less calcium and phosphorus and more sulfur than the neighboring bone lamellae. Most authors assume that the cement line is impermeable, while others assume that some canaliculi are crossing the cement line which will make it permeable to certain degree. The objective of this work is to develop a theoretical analysis to study the leakage through the cement line and its relationship with the pore pressure distribution. The theoretical analysis is developed using our previous analysis for osteon under harmonic loading with addition of leakage parameter. The leakage parameter varies from 0 to 1 , where a value of 0 indicates free flow through the cement line and a value of 1 indicates $n o$ flow through the cement line. Experimental results could be compared to this developed theoretical solution to get in depth understanding of the effect of leakage on osteon poroelastic properties. Additionally, the developed theoretical solution will give insight into sensitivity of osteon pore pressure to leakage through the cement line.
\end{abstract}

Keywords: Cement line, osteon permeability, osteon leakage

\section{Introduction}

\subsection{Bone microstructure}

Any attempts to understand and model the biomechanical properties of bone must be firmly rooted in knowledge of the structure of bone on both the ultrastructural and microstructural levels of organization (Figure 1). Mechanical properties of bone are determined by a multiplicity of materials and structural properties such as tissue mineralization, size and composition of mineral crystals, anisotropy ...etc. Time varying mechanical loads applied to bone generates fluid pressure gradients in the lacunar canalicular porosity (PLC) that contributes to the interstitial fluid flow and the transport in the PLC. The interstitial fluid flow is important for cellular nutrition and waste removal, and it is a critical factor in osteocyte mechanotransduction.

The pore sizes in cortical bone are approximately three discrete sizes; the largest pore size (approximately $50 \mu \mathrm{m}$ diameter) is associated with the vascular porosity (PV), the second largest pore size (approximately $0.3 \mu \mathrm{m}$ diameter) with the canaliculi in the lacunar-canalicular porosity (PLC) and the smallest pore size (approximately $10 \mathrm{~nm}$ diameter) is the collagen-apatite porosity 
(PCA). The typical pore size of the vascular porosity channels is not the blood vessel pore size; rather it is the size of the tubular tunnels (osteonal systems or osteons and Volkmann canals) containing the blood vessels, the arterioles and the venules, and considerable extra pore fluid space that contains no blood vessels. The interstitial pore fluid in the collagen-apatite porosity (PCA) is considered to be bound to the solid structure and the PCA is not of interest in the present considerations. Thus, the two pore size structures of interest in the flow of bone pore fluid are the vascular porosity (PV), and the lacunar-canalicular porosity (PLC) - osteons; the ratio of the pore sizes is approximately 167 . Osteons can be found in long bones of some animals and all humans. Osteons are small in size and thus are challenging to isolate ${ }^{1,2,3,4}$.

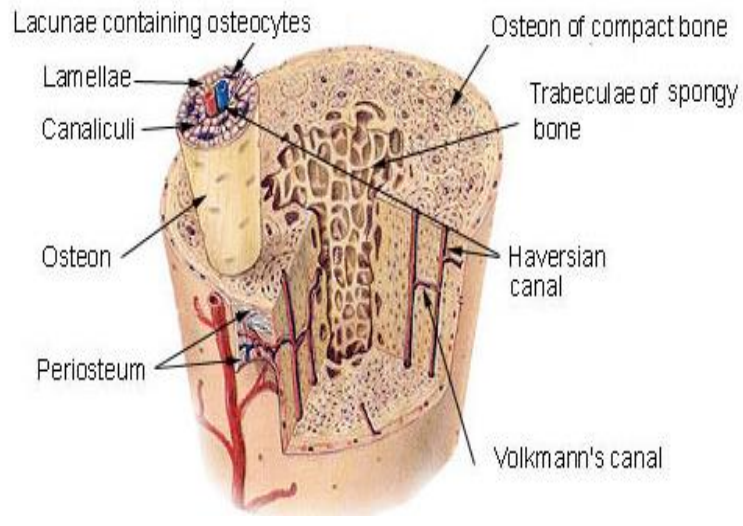

Figure. 1. A cartoon of the midsection of a long bone with one of the many osteons ( 250 micrometers in diameter approximately) highlighted for illustration. Both the midsection of the long bone and the osteon are idealized as hollow circular cylinders. The hollow zone of the smaller cylinder is the osteonal or the Haversian canal. The interior of the hollow circular cylinder contains the PLC.

\subsection{The cement line}

The cement line can be described as the outer domain of the osteon. Researchers suggested that the cement line is less highly mineralized and produced evidence that it has less calcium phosphorus and more sulfur than the neighboring bone lamellae ${ }^{5}$. However, there is an agreement that there is less collagen in it. Osteon diameter in average is 250 micrometers. Canaliculi are passage ways between the cells that cover the osteonal canal and the lacunae. Viscous-like motion at cement lines between osteons might be responsible for a portion of the viscoelasticity in bone particularly at long times and low frequencies ${ }^{6}$ as ascertained experimentally in bovine bone ${ }^{7}$ and in human bones ${ }^{8}$. It is not easy to determine whether the boundary formed by the cement line is permeable or not. However, many authors assumed that the cement line is impermeable ${ }^{9,10}$. Some recent evidence shows that some canaliculi cross the cement line. These crossings conflict with the assumption of impermeability. The canaliculi might be crossing where there is an isolated osteocyte on the other side, as a result of bone remodeling. Also, the canaliculi might be crossing to communicate with a neighboring osteon to complete a whole bone cell network. An earlier theoretical study by Wang ${ }^{11}$ 
concluded that it made little difference if the boundary is_assumed to be impermeable or fully permeable in the global prediction of physiological significance.

\subsection{The Unconfined Compression}

The problem of unconfined compression of porous disks compressed between two parallel rigid and impermeable smooth plates in order to determine the soft tissue's permeability and some of its elastic constants has been under investigation by many authors in the last few decades ${ }^{12,13,14,15}$. The impermeable frictionless plates would allow flow only in the radial direction in the unconfined compression experiment. The specimen contact with the plates is assumed to be frictionless, so the deformation is expected to be independent of z. Typical unconfined compression tests are accomplished by imposing either a step in end plate displacement, which would constitute a stress relaxation test, or a step in total applied end force load may be applied, which would constitute a creep test.

\section{Materials and Methods}

For the analysis of soft materials the assumption that the fluid and solid constituents of the poroelastic medium are both incompressible is reasonable. However, in the case of hard biological tissues the constituent incompressibility assumption is no longer reasonable because the effective bulk modulus of the poroelastic solid constituent is almost an order of magnitude stiffer than that of the poroelastic fluid constituent, thus the solid constituent shields the fluid constituent from stress. Therefore, compressibility will be assumed. Transverse isotropy appears to be more realistic in modeling deformation of porous cylindrical disks and many authors have used it successfully. The analytical approach (see Ref. 16) derived a detailed solution for three types of annular porous disks under unconfined compression based on their boundary conditions. These boundary conditions are: (i) free fluid passage across the outer and inner cylindrical boundaries of the disk; (ii) free fluid passage across the outer cylindrical boundary and no flow across the inner boundary (i.e. zero pressure gradient); (iii) free fluid passage across the inner cylindrical boundary and no flow across the outer boundary. The first (i) and the third (iii) boundary conditions will fit nicely into our investigation of the cement line permeability. Curve fitting the boundary conditions (i) and (iii) to the experimental testing of the two osteon types will give good insight into the cement line permeability.

The general formulation of the mathematical problem for compressible poroelastic hollow circular cylinders has been described earlier ${ }^{16,17,18}$. The porosity level is designated as $\mathrm{L}$ as shorthand for PLC. The formulation is based on the following assumptions concerning the functional dependence of variables: cylindrical coordinates are employed and axial symmetry is assumed, and displacement field components $\left(u_{r}^{L}, u_{z}^{L}\right)$ the pressure $p^{L}$ and the variation in fluid content $\xi^{L}$ are assumed to have the functional dependencies indicated below (see Ref. 17, Eq. (47), Ref. 16, Eq. (1))

$$
u_{r}^{L}=u^{L}(r, t), u_{z}^{L}=z \varepsilon(t), p^{L}=p^{L}(r, t), \zeta^{L}=\zeta^{L}(r, t),
$$

where $\varepsilon(t)$ is the applied axial loading that is $\varepsilon(t)=\varepsilon_{o} e^{i \omega t}$ in this case. Thus the key variables are taken to be $u^{L}(r, t), p^{L}(r, t)$ and $\left.\zeta^{L}(r, t)\right)$ and the solutions are assumed to be of the form. 


$$
\begin{aligned}
& u_{r}^{L}(r, t)=u^{L \omega}(r) e^{i \omega t}, u_{z}^{L}(t)=z \varepsilon_{o} e^{i \omega t}, \\
& p^{L}(r, t)=p^{L \omega}(r) e^{i \omega t}, \zeta^{L}(r, t)=\zeta^{L \omega}(r) e^{i \omega t},
\end{aligned}
$$

the specific solutions sought are for $u^{L \omega}(r), p^{L \omega}(r)$, and $\left.\zeta^{L \omega}(r)\right)$. The non-zero stress components $T_{r r}, T_{\theta \theta}$ and $T_{z z}$ are determined to be (see Ref. 17, Eq. (60), Ref. 16, Eq. (20))

$$
\begin{aligned}
& T_{r r}=-\hat{A}_{r}^{L} p^{L}+\hat{C}_{r r}^{d, L} \frac{\partial u^{L}}{\partial r}+\hat{C}_{r \theta}^{d, L} \frac{u^{L}}{r}+\hat{C}_{r z}^{d, L} \varepsilon_{o} e^{i \omega t} \\
& T_{\theta \theta}=-\hat{A}_{r}^{L} p^{L}+\hat{C}_{r \theta}^{d, L} \frac{\partial u^{L}}{\partial r}+\hat{C}_{r r}^{d, L} \frac{u^{L}}{r}+\hat{C}_{r z}^{d, L} \varepsilon_{o} e^{i \omega t}, \\
& T_{z z}=-\hat{A}_{z}^{L} p^{L}+\hat{C}_{r z}^{d, L}\left(\frac{\partial u^{L}}{\partial r}+\frac{u^{L}}{r}\right)+\hat{C}_{z z}^{d, L} \varepsilon_{o} e^{i \omega t}
\end{aligned}
$$

where the components of $\hat{\mathbf{C}}^{d, L}$ are the components of the drained elasticity tensor at the PLC porosity level and $\hat{\mathbf{A}}^{L}$ are those of the Biot effective stress coefficient vector at the PLC porosity level. The values of these coefficients and others used in demonstrating the results in this paper are given in Table 1. Table 2 contains formulas relating the various coefficients used in the calculations that follow.

Table 1: The material properties reported from different sources for the elastic moduli, Poisson's ratios...etc. The superscript $d$ reflects the drained properties and the superscript $m$ reflects the matrix material properties. In the text the PLC is referred to by the superscript L. The water compressibility $\left(K_{f}\right)$ is $2.3 \mathrm{GPa}$, its viscosity $\mu$ is $0.001 \mathrm{~Pa}$.s, and the amplitude of $\dot{\varepsilon}_{0}$, the applied strain is $0.0001^{17}$.

\begin{tabular}{|ll|}
\hline Material Parameters & PLC (L) \\
$E_{1}^{d}=E_{2}^{d}$ & $15.17 \mathrm{GPa}$ \\
$E_{3}^{d}$ & $15.96 \mathrm{GPa}$ \\
$v_{12}^{d}=v_{21}^{d}$ & 0.316 \\
$v_{31}^{d}=v_{32}^{d}$ & 0.308 \\
$v_{13}^{d}=v_{23}^{d}$ & 0.282 \\
$E_{1}^{m}=E_{2}^{m}$ & $18.6 \mathrm{GPa}$ \\
$E_{3}^{m}$ & $22.32 \mathrm{GPa}$ \\
$v_{12}^{m}=v_{21}^{m}$ & 0.322 \\
$v_{31}^{m}=v_{32}^{m}$ & 0.312 \\
$v_{13}^{m}=v_{23}^{m}$ & 0.255 \\
\hline
\end{tabular}




\begin{tabular}{|ll|}
\hline outer radius (osteon) & $r_{\mathrm{o}}=100 \mathrm{\mu m}$ \\
inner radius (osteon) & $r_{i}=20 \mu \mathrm{m}$ \\
porosity $\phi$ & 0.05 \\
permeability $K_{r r}$ & $2.2 \times 10^{-19} \mathrm{~m}^{2}$ \\
\hline
\end{tabular}

Table 2. The values of various bulk moduli and constants defined for convenience, for case of transverse isotropy compressible material model ${ }^{17}$.

\begin{tabular}{|c|c|}
\hline & Transverse isotropy compressible \\
\hline 1 & $3\left(1-v_{12}^{m}-v_{13}^{m}\right)$ \\
\hline$K_{1}^{m}$ & $E_{1}^{m}$ \\
\hline 1 & $\underline{3\left(1-2 v_{13}^{m}\right)}$ \\
\hline$K_{3}^{m}$ & $E_{3}^{m}$ \\
\hline 1 & $2+1$ \\
\hline$\overline{K_{\mathrm{R}_{\text {eff }}}^{m}}$ & $\overline{3 K_{1}^{m}}+\overline{3 K_{3}^{m}}$ \\
\hline 1 & $\underline{2\left(1-v_{12}^{d}-v_{13}^{d}\right)}+\frac{1-2 v_{31}^{d}}{2}$ \\
\hline$K_{\mathrm{R}_{\mathrm{eff}}}^{d}$ & $E_{1}^{d}$ \\
\hline$C_{e f f}^{d}$ & $\frac{1}{-}-\frac{1}{}+\phi\left(\frac{1}{-}-\frac{1}{-}\right)$ \\
\hline & $K_{\mathrm{R}_{\text {eff }}}^{d} \quad K_{\mathrm{R}_{\text {eff }}}^{m} \quad K^{f} \quad K_{\mathrm{R}_{\text {eff }}}^{m}$ \\
\hline$\Lambda$ & $C^{d}-\frac{1}{2}+\frac{2}{-1}\left[2 E_{1}^{d}+\frac{4 E_{1}^{d} v_{31}^{d}}{E^{d}}+\frac{E_{3}^{d}\left(1-v_{12}^{d}\right)}{1}\right.$ \\
\hline & 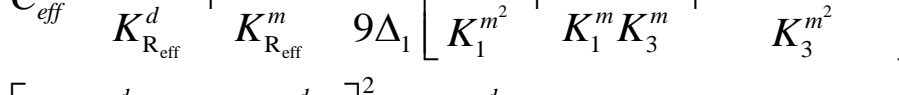 \\
\hline$J$ & {$\left[1-\frac{E_{1}^{d}}{3 \Delta_{1}}\left(\frac{1}{K_{1}^{m}}+\frac{v_{31}^{d}}{K_{3}^{m}}\right)\right]^{2}+\Lambda \frac{E_{1}^{d} \Delta_{2}}{\Delta_{1}}$} \\
\hline$\hat{A}_{r}$ & $1-\frac{E_{1}^{d}}{3 \Delta_{1}}\left(\frac{1}{K_{1}^{m}}+\frac{v_{31}^{d}}{K_{3}^{m}}\right)$ \\
\hline$\hat{A}_{z}$ & $1-\frac{E_{3}^{d}}{3 \Delta_{1}}\left(\frac{2 v_{13}^{d}}{K_{1}^{m}}+\frac{1-v_{12}^{d}}{K_{3}^{m}}\right)$ \\
\hline
\end{tabular}

The only equation of equilibrium that is not satisfied automatically is (see Ref. 17, Eq. (61), Ref. 16, Eq. (21))

$$
\frac{\partial T_{r r}}{\partial r}+\frac{T_{r r}-T_{\theta \theta}}{r}=0
$$


Substitution of the non-zero stress components (3) into the equilibrium equation (4) yields a result that is subsequently integrated with respect to $r$ to produce the following expression:

$$
\frac{\partial u^{L}}{\partial r}+\frac{u^{L}}{r}=\frac{1}{r} \frac{\partial}{\partial r}\left(r u^{L}\right)=\frac{\hat{A}_{r}^{L}}{\hat{C}_{r r}^{d, L}} p^{L}(r, t)+f_{o}^{L \omega} e^{i \omega t},
$$

where $f_{o}^{L \omega} e^{i \omega t}$ is a function of time obtained in the integration with respect to $\mathrm{r}$ and $\hat{A}_{r}^{L}$ is the $r$ component of the Biot effective stress coefficient. Since both the functions $u^{L}(r, t)$ and $p^{L}(r, t)$ in (1) are of the form of a product of a function of $r$ times $e^{i \omega t}$ we assume that the function of time obtained in the integral has the representation $f_{o}^{L \omega} e^{i \omega t}$. The expression for $\frac{\partial u^{L}}{\partial r}+\frac{u^{L}}{r}=\frac{1}{r} \frac{\partial}{\partial r}\left(r u^{L}\right)$ may be used to calculate two expressions for the quantity $\hat{\mathbf{A}}^{L} \cdot \hat{\mathbf{E}}$,

$$
\hat{\mathbf{A}}^{L} \cdot \hat{\mathbf{E}}=\hat{A}_{r}^{L}\left(\frac{\partial u^{L}}{\partial r}+\frac{u^{L}}{r}\right)+\hat{A}_{z}^{L} \varepsilon_{o} e^{i \omega t}
$$

(see Ref. 17, Eq. (59), Ref. 16, Eq. (23)), one in terms of the variation in fluid content $\xi^{L}$ and the other in terms of the pressure $p^{L}$. These expressions are needed in the diffusion equations (20) and (22) for $\xi^{L}$ and $p^{L}$, respectively ${ }^{18}$. To find $\hat{\mathbf{A}}^{L} \cdot \hat{\mathbf{E}}$ in terms of $p^{L}$ substitute (5) in (6) to obtain

$$
\hat{\mathbf{A}}^{L} \cdot \hat{\mathbf{E}}=\frac{\hat{A}_{r}^{L} \hat{A}_{r}^{L}}{\hat{C}_{r r}^{d p}} p^{L}(r, t)+\left(\hat{A}_{z}^{L} \varepsilon_{o}+\hat{A}_{r}^{L} f_{o}^{L \omega}\right) e^{i \omega t}
$$

To find $\hat{\mathbf{A}}^{L} \cdot \hat{\mathbf{E}}$ eliminate $p^{L}$ in (7) using eq. (7) in Ref. 18 with $P$ replaced by $L$, thus

$$
\hat{\mathbf{A}}^{L} \cdot \hat{\mathbf{E}}=\frac{1}{J^{L}}\left\{\hat{A}_{r}^{L} \hat{A}_{r}^{L} \zeta^{L}+\Lambda^{L} \hat{C}_{r r}^{d, L}\left[\hat{A}_{z}^{L} \varepsilon_{o}+\hat{A}_{r}^{L} f_{o}^{L \omega}\right] e^{i \omega t}\right\}
$$

where the dimensionless quantity $J^{L}$ is introduced,

$$
J^{L}=\hat{A}_{r}^{L} \hat{A}_{r}^{L}+\Lambda^{L} \hat{C}_{r r}^{d, L},
$$

and

$$
\Lambda^{L}=C_{e f f}^{d, L}-\frac{1}{K_{R e f f}^{d, L}}+\frac{2}{K_{R e f f}^{m}}-\frac{1}{\left(1-v_{12}^{d, L}-2 v_{13}^{d, L} v_{31}^{d, L}\right)}\left[\frac{2 E_{1}^{d, L}}{\left(K_{1}^{m}\right)^{2}}+\frac{4 E_{1}^{d, L} v_{31}^{d, L}}{K_{1}^{m} K_{3}^{m}}+\frac{E_{3}^{d, L}\left(1-v_{12}^{d, L}\right)}{\left(K_{3}^{m}\right)^{2}}\right],
$$

and 


$$
C_{\text {eff }}^{d, L}=\frac{1}{K_{\text {Reff }}^{d, L}}-\frac{1}{K_{\text {Reff }}^{m}}+\phi\left(\frac{1}{K^{f}}-\frac{1}{K_{\text {Reff }}^{m}}\right)
$$

which are Eqs. (66) and (19C) in Ref. (17) - There is an error in equations (66) in Ref. (17) that is corrected in equation (10) above: specifically the last term in Eq. (33) is divided by 9 in Ref. 17. In the equations above the kernel letter $K$ stands for a bulk modulus, the superscripts $d$ and $f$ indicate drained material and the fluid, respectively, $m$ indicates the matrix material and the subscript Reff indicates the effective Reuss lower bound on the indicated bulk moduli. In the compressible,

transversely anisotropic case, the expression for $J^{L}$ is given by (see Ref. 17, Eq. (57)),

$$
J^{L}=\left[1-\frac{E_{1}^{d, L}}{3\left(1-v_{12}^{d, L}-2 v_{31}^{d, L} v_{13}^{d, L}\right)}\left(\frac{1}{K_{1}^{M}}+\frac{v_{31}^{d, L}}{K_{3}^{M}}\right)\right]^{2}+\Lambda^{L} \frac{E_{1}^{d, L}\left(1-v_{13}^{d, L} v_{31}^{d, L}\right)}{\left(1+v_{12}^{d, L}\right)\left(1-v_{12}^{d, L}-2 v_{31}^{d, L} v_{13}^{d, L}\right)}
$$

The diffusive differential equation for the pore pressure is obtained by substituting Eq. (7) and $\hat{\mathbf{K}}^{L} \cdot \hat{\mathbf{O}}=K_{r r}^{L}\left(\frac{\partial^{2}}{\partial r^{2}}+\frac{1}{r} \frac{\partial}{\partial r}\right)$ into Eq. (22) in Ref. 17, neglecting the flux terms from the other porosity level, thus

$$
\frac{\partial p^{L}}{\partial t}-\frac{K_{r r}^{L}}{\mu \Lambda^{L}}\left(\frac{\partial^{2} p^{L}}{\partial r^{2}}+\frac{1}{r} \frac{\partial p^{L}}{\partial r}\right)=-\frac{1}{\Lambda^{L}} \frac{\hat{A}_{r}^{L} \hat{A}_{r}^{L}}{\hat{C}_{r r}^{d, L}} \frac{\partial p^{L}}{\partial t}-\frac{i \omega}{\Lambda^{L}}\left(\hat{A}_{z}^{L} \varepsilon_{o}+\hat{A}_{r}^{L} f_{o}^{L \omega}\right) e^{i \omega t}
$$

or, rearranging terms,

$$
\frac{\partial^{2} p^{L}}{\partial r^{2}}+\frac{1}{r} \frac{\partial p^{L}}{\partial r}-\frac{\mu}{K_{r r}^{L}}\left(\Lambda^{L}+\frac{\hat{A}_{r}^{L} \hat{A}_{r}^{L}}{\hat{C}_{r r}^{d, L}}\right) \frac{\partial p^{L}}{\partial t}=\frac{i \omega \mu}{K_{r r}^{L}}\left(\hat{A}_{z}^{L} \varepsilon_{o}+\hat{A}_{r}^{L} f_{o}^{L \omega}\right) e^{i \omega t}
$$

and introducing the notation

$$
\frac{1}{c^{L}}=\frac{\mu}{K_{r r}^{L}}\left(\Lambda^{L}+\frac{\hat{A}_{r}^{L} \hat{A}_{r}^{L}}{\hat{C}_{r r}^{d, L}}\right)=\frac{\mu J^{L}}{K_{r r}^{L} \hat{C}_{r r}^{d, L}}
$$

where Eq. (9) has been employed. It follows that

$$
\frac{\partial^{2} p^{L}}{\partial r^{2}}+\frac{1}{r} \frac{\partial p^{L}}{\partial r}-\frac{1}{c^{L}} \frac{\partial p^{L}}{\partial t}=\frac{i \omega \mu}{K_{r r}^{L}}\left(\hat{A}_{z}^{L} \varepsilon_{o}+\hat{A}_{r}^{L} f_{o}^{L \omega}\right) e^{i \omega t}
$$

Introducing the dimensionless radius $\lambda=r / r_{o}$, the representation $p^{L}(r, t)=p^{L \omega}(r) e^{i \omega t}$ from Eq. (2), and the dimensionless driving frequencies constant $\bar{\omega}$ as well as a constant of dimension stress $\Upsilon^{L}$,

$$
, \bar{\omega}=\frac{\omega r_{o}^{2}}{c^{L}}, \Upsilon^{L}=\frac{\mu \hat{A}_{r}^{L} c^{L}}{K_{r r}^{L}}=\frac{\hat{A}_{r}^{L} \hat{C}_{r r}^{d, L}}{J^{L}} .
$$


The differential equation takes the form

$$
\left\{\frac{\partial^{2} p^{L \omega}}{\partial \lambda^{2}}+\frac{1}{\lambda} \frac{\partial p^{L \omega}}{\partial \lambda}-i \bar{\omega} p^{L \omega}-i \bar{\omega} r^{L}\left(\frac{\hat{A}_{z}^{L}}{\hat{A}_{r}^{L}} \varepsilon_{o}+f_{o}^{L \omega}\right)\right\} e^{i \omega t}=0
$$

The solution to this equation is

$$
p^{L}(\lambda, t)=\left\{A_{1}^{\omega} I_{o}(\sqrt{i \bar{\omega}} \lambda)+A_{2}^{\omega} K_{o}(\sqrt{i \bar{\omega}} \lambda)-\Upsilon^{L} \frac{\hat{A}_{z}^{L}}{\hat{A}_{r}^{L}} \varepsilon_{o}-\Upsilon^{L} f_{o}^{L \omega}\right\} e^{i \omega t}
$$

Where $I_{0}(x)$ and $K_{0}(x)$ are modified Bessel functions of the first and second type respectively. Here we introduce the leakage parameter $\eta$ which we will use to control the flow through the cement line. The range of the leakage parameter is

$$
0 \leq \eta \leq 1
$$

Where the value of zero indicates free flow through the cement line, and the value of 1 indicates no flow through the cement line. Thus, the boundary conditions can be expressed as

$$
\begin{gathered}
p^{L}(a, t)=0 \\
\eta \frac{\partial p^{L}}{\partial \lambda}(1, t)+(1-\eta) p^{L}(1, t)=0
\end{gathered}
$$

Where $a$ is the non-dimensional inner radius of the osteon. Eq. (21) indicates free flow through the Haversian canal (inner walls), while Eq. (22) indicates different degrees of flow based on the value of the leakage parameter.

Substitute the first boundary condition

$$
p^{L}(a, t)=0=\left(A_{1}^{\omega} I_{o}(\sqrt{i \bar{\omega}} a)+A_{2}^{\omega} K_{o}(\sqrt{i \bar{\omega}} a)-\Upsilon^{L} \frac{\hat{A}_{z}^{L}}{\hat{A}_{r}^{L}} \varepsilon_{o}-\Upsilon^{L} f_{o}^{L \omega}\right)
$$

From which

$$
A_{1}^{\omega}=\frac{\left(\Upsilon^{L}\left[\frac{\hat{A}_{z}^{L}}{\hat{A}_{r}^{L}} \varepsilon_{o}+f_{o}^{L \omega}\right]-A_{2}^{\omega} K_{o}(\sqrt{i \overline{\bar{\omega}} a})\right)}{I_{o}(\sqrt{i \bar{\omega}} a)}
$$

Second boundary condition:

$$
\frac{\partial p^{L}}{\partial \lambda}(1, t)=\left\{\sqrt{i \bar{\omega}} A_{1}^{\omega} I_{1}(\sqrt{i \bar{\omega}})-A_{2}^{\omega} \sqrt{i \bar{\omega}} K_{1}(\sqrt{i \bar{\omega}})\right\} e^{i \omega t}
$$




$$
\begin{gathered}
p^{L}(1, t)=\left\{A_{1}^{\omega} I_{o}(\sqrt{i \bar{\omega}})+A_{2}^{\omega} K_{o}(\sqrt{i \bar{\omega}})-\Upsilon^{L} \frac{\hat{A}_{z}^{L}}{\hat{A}_{r}^{L}} \varepsilon_{o}-\Upsilon^{L} f_{o}^{L \omega}\right\} e^{i \omega t} \\
\eta \frac{\partial p^{L}}{\partial \lambda}(1, t)+(1-\eta) p^{L}(1, t)=0=\eta\left\{\sqrt{i \bar{\omega}} A_{1}^{\omega} I_{1}(\sqrt{i \bar{\omega}})-A_{2}^{\omega} \sqrt{i \bar{\omega}} K_{1}(\sqrt{i \bar{\omega}})\right\} \\
+(1-\eta)\left(A_{1}^{\omega} I_{o}(\sqrt{i \bar{\omega}})+A_{2}^{\omega} K_{o}(\sqrt{i \bar{\omega}})-\Upsilon^{L} \frac{\hat{A}_{z}^{L}}{\hat{A}_{r}^{L}} \varepsilon_{o}-\Upsilon^{L} f_{o}^{L \omega}\right)
\end{gathered}
$$

From which we conclude,

$$
\begin{aligned}
& 0=A_{1}^{\omega}\left[\eta \sqrt{i \bar{\omega}} I_{1}(\sqrt{i \bar{\omega}})+I_{o}(\sqrt{i \bar{\omega}})-\eta I_{o}(\sqrt{i \bar{\omega}})\right]- \\
& A_{2}^{\omega}\left[\eta \sqrt{i \bar{\omega}} K_{1}(\sqrt{i \bar{\omega}})-K_{o}(\sqrt{i \bar{\omega}})+\eta K_{o}(\sqrt{i \bar{\omega}})\right]-\Upsilon^{L} \frac{\hat{A}_{z}^{L}}{\hat{A}_{r}^{L}} \varepsilon_{o} 1-\eta-\Upsilon^{L} f_{o}^{L \omega} 1-\eta
\end{aligned}
$$

Substitute Eq. (24) into the first term of Eq. (25)

$$
A_{1}^{\omega} \mathrm{X}_{1}(\bar{\omega}: \eta)=A_{2}^{\omega} \mathrm{X}_{2}(\bar{\omega}: \eta)+\Upsilon^{L} 1-\eta\left(\frac{\hat{A}_{z}^{L}}{\hat{A}_{r}^{L}} \varepsilon_{o}+f_{o}^{L \omega}\right)
$$

where

$$
\mathrm{X}_{1}(\bar{\omega}: \eta)=\eta \sqrt{i \bar{\omega}} I_{1}(\sqrt{i \bar{\omega}})+I_{o}(\sqrt{i \bar{\omega}})-\eta I_{o}(\sqrt{i \bar{\omega}})
$$$$
\mathrm{X}_{2}(\bar{\omega}: \eta)=\eta \sqrt{i \bar{\omega}} K_{1}(\sqrt{i \bar{\omega}})-K_{o}(\sqrt{i \bar{\omega}})+\eta K_{o}(\sqrt{i \bar{\omega}})
$$

This will lead to,

$$
A_{2}^{\omega}=\frac{\Upsilon^{L}\left[\frac{\hat{A}_{z}^{L}}{\hat{A}_{r}^{L}} \varepsilon_{o}+f_{o}^{L \omega}\right] \mathrm{X}_{1}(\bar{\omega}: \eta)-1-\eta I_{o}(\sqrt{i \bar{\omega}} a)}{\mathrm{X}_{2}(\bar{\omega}: \eta) I_{o}(\sqrt{i \bar{\omega}} a)+K_{o}(\sqrt{i \bar{\omega}} a) \mathrm{X}_{1}(\bar{\omega}: \eta)}
$$

For the radial displacement,

$$
\begin{aligned}
& \frac{1}{\lambda} \frac{\partial}{\partial \lambda}\left(\lambda u^{L}\right)=r_{o} \frac{\hat{A}_{r}^{L}}{\hat{C}_{r r}^{d, L}} p^{L}(\lambda, t)+r_{o} f_{o}^{L \omega} e^{i \omega t} \\
& \frac{\partial}{\partial \lambda}\left(\lambda u^{L}\right)=r_{o} \frac{\hat{A}_{r}^{L}}{\hat{C}_{r r}^{d, L}} \\
& \left(\left\{A_{1}^{\omega} \lambda I_{o}(\sqrt{i \bar{\omega}} \lambda)+A_{2}^{\omega} \lambda K_{o}(\sqrt{i \bar{\omega}} \lambda)-\Upsilon^{L} \frac{\hat{A}_{z}^{L}}{\hat{A}_{r}^{L}} \varepsilon_{o} \lambda-\Upsilon^{L} f_{o}^{L \omega} \lambda\right\} e^{i \omega t}\right)+r_{o} f_{o}^{L \omega} \lambda e^{i \omega t}
\end{aligned}
$$

By integration, 


$$
\begin{aligned}
& u^{L}=r_{o} \frac{\hat{A}_{r}^{L}}{\hat{C}_{r r}^{d, L}}\left(\left\{A_{1}^{\omega} \frac{I_{1}(\sqrt{i \bar{\omega}} \lambda)}{\sqrt{i \bar{\omega}}}-A_{2}^{\omega} \frac{K_{1}(\sqrt{i \bar{\omega}} \lambda)}{\sqrt{i \bar{\omega}}}-\Upsilon^{L} \frac{\hat{A}_{z}^{L}}{2 \hat{A}_{r}^{L}} \varepsilon_{o} \lambda-\Upsilon^{L} f_{o}^{L \omega} \frac{\lambda}{2}\right\} e^{i \omega t}\right) \\
& +r_{o} f_{o}^{L \omega} \frac{\lambda}{2} e^{i \omega t}+\frac{c}{\lambda} e^{i \omega t}
\end{aligned}
$$

Where $\mathrm{c}$ is the constant of integration.

The radial stresses

$$
T_{r r}=-\hat{A}_{r}^{L} p^{L}(\lambda, t)+\hat{C}_{r r}^{d, L} \frac{1}{r_{o}}\left[r_{o} \frac{\hat{A}_{r}^{L}}{\hat{C}_{r r}^{d, L}} p^{L}(\lambda, t)+r_{o} f_{o}^{L \omega} e^{i \omega t}\right]+\left(\hat{C}_{r \theta}^{d, L}-\hat{C}_{r r}^{d, L}\right) \frac{u^{L}}{r_{o} \lambda}+\hat{C}_{r z}^{d, L} \varepsilon_{o} e^{i \omega t}
$$

Requiring in the inner boundaries the radial stresses to vanish will lead to

$$
T_{r r}=0=\hat{C}_{r r}^{d, L}\left[f_{o}^{L \omega} e^{i \omega t}\right]+\left(\hat{C}_{r \theta}^{d, L}-\hat{C}_{r r}^{d, L}\right) \frac{u^{L}}{r_{o} a}+\hat{C}_{r z}^{d, L} \varepsilon_{o} e^{i \omega t}
$$

Substitution of the radial displacement into the equation above,

$$
\begin{aligned}
& 0=\hat{C}_{r r}^{d, L} f_{o}^{L \omega}+ \\
& \frac{\left(\hat{C}_{r \theta}^{d, L}-\hat{C}_{r r}^{d, L}\right)}{r_{o} a}\left[r_{o} \frac{\hat{A}_{r}^{L}}{\hat{C}_{r r}^{d, L}}\left(\begin{array}{l}
\left\{A_{1}^{\omega} \frac{I_{1}(\sqrt{i \bar{\omega}} a)}{\sqrt{i \bar{\omega}}}-A_{2}^{\omega} \frac{K_{1}(\sqrt{i \bar{\omega}} a)}{\sqrt{i \bar{\omega}}}-\right. \\
\left.\Upsilon^{L} \frac{\hat{A}_{z}^{L}}{2 \hat{A}_{r}^{L}} \varepsilon_{o} a-\Upsilon^{L} f_{o}^{L \omega} \frac{a}{2}\right\}
\end{array}\right]+r_{o} f_{o}^{L \omega} \frac{a}{2}+\frac{c}{a}\right]+\hat{C}_{r z}^{d, L} \varepsilon_{o}
\end{aligned}
$$

Now we require the effective stresses at the outer boundary (the cement line) is equal to zero

$$
T_{r r}+\hat{A}_{r}^{L} p^{L}=0
$$

Which can be expanded and simplified

$$
\hat{C}_{r r}^{d, L}\left[\frac{\hat{A}_{r}^{L}}{\hat{C}_{r r}^{d, L}} p^{L}(1, t)+f_{o}^{L \omega} e^{i \omega t}\right]+\left(\hat{C}_{r \theta}^{d, L}-\hat{C}_{r r}^{d, L}\right) \frac{u^{L}(1, t)}{r_{o}}+\hat{C}_{r z}^{d, L} \varepsilon_{o} e^{i \omega t}=0
$$

Pore pressure and radial displacement in the outer boundaries can be specified from Eqs. (19) and (28)

$$
p^{L}(1, t)=\left\{A_{1}^{\omega} I_{o}(\sqrt{i \bar{\omega}})+A_{2}^{\omega} K_{o}(\sqrt{i \bar{\omega}})-\Upsilon^{L} \frac{\hat{A}_{z}^{L}}{\hat{A}_{r}^{L}} \varepsilon_{o}-\Upsilon^{L} f_{o}^{L \omega}\right\} e^{i \omega t}
$$




$$
\begin{aligned}
& u^{L}(1, t)=r_{o} \frac{\hat{A}_{r}^{L}}{\hat{C}_{r r}^{d, L}}\left(\left\{A_{1}^{\omega} \frac{I_{1}(\sqrt{i \bar{\omega}})}{\sqrt{i \bar{\omega}}}-A_{2}^{\omega} \frac{K_{1}(\sqrt{i \bar{\omega}})}{\sqrt{i \bar{\omega}}}-\Upsilon^{L} \frac{\hat{A}_{z}^{L}}{2 \hat{A}_{r}^{L}} \varepsilon_{o}-\frac{1}{2} \Upsilon^{L} f_{o}^{L \omega}\right\} e^{i \omega t}\right) \\
& +\frac{1}{2} r_{o} f_{o}^{L \omega} e^{i \omega t}+c e^{i \omega t}
\end{aligned}
$$

Set

$$
\Gamma(\bar{\omega})=A_{1}^{\omega} I_{o}(\sqrt{i \bar{\omega}})+A_{2}^{\omega} K_{o}(\sqrt{i \bar{\omega}})
$$

Thus

$$
p^{L}(1, t)=\left(\Gamma(\bar{\omega})-\Upsilon^{L} \frac{\hat{A}_{z}^{L}}{\hat{A}_{r}^{L}} \varepsilon_{o}-\Upsilon^{L} f_{o}^{L \omega}\right) e^{i \omega t}
$$

Also set

$$
\Theta(\bar{\omega})=A_{1}^{\omega} \frac{I_{1}(\sqrt{i \bar{\omega}})}{\sqrt{i \bar{\omega}}}-A_{2}^{\omega} \frac{K_{1}(\sqrt{i \bar{\omega}})}{\sqrt{i \bar{\omega}}}
$$

Thus

$$
u^{L}(1, t)=r_{o} \frac{\hat{A}_{r}^{L}}{\hat{C}_{r r}^{d, L}}\left(\left\{\Theta(\bar{\omega})-\Upsilon^{L} \frac{\hat{A}_{z}^{L}}{2 \hat{A}_{r}^{L}} \varepsilon_{o}-\frac{1}{2} \Upsilon^{L} f_{o}^{L \omega}\right\} e^{i \omega t}\right)+\frac{1}{2} r_{o} f_{o}^{L \omega} e^{i \omega t}+c e^{i \omega t}
$$

Rewrite Eq. (29),

$$
\begin{aligned}
& T_{r r}+\hat{A}_{r}^{L} p^{L}=0= \\
& \hat{C}_{r r}^{d, L}\left[\frac{\hat{A}_{r}^{L}}{\hat{C}_{r r}^{d, L}}\left(\Gamma(\bar{\omega})-\Upsilon^{L} \frac{\hat{A}_{z}^{L}}{\hat{A}_{r}^{L}} \varepsilon_{o}-\Upsilon^{L} f_{o}^{L \omega}\right) e^{i \omega t}+f_{o}^{L \omega} e^{i \omega t}\right]+ \\
& \left(\hat{C}_{r \theta}^{d, L}-\hat{C}_{r r}^{d, L}\right) \frac{r_{o} \frac{\hat{A}_{r}^{L}}{\hat{C}_{r r}^{d, L}}\left(\left\{\Theta(\bar{\omega})-\Upsilon^{L} \frac{\hat{A}_{z}^{L}}{2 \hat{A}_{r}^{L}} \varepsilon_{o}-\frac{1}{2} \Upsilon^{L} f_{o}^{L \omega}\right\} e^{i \omega t}\right)+\frac{1}{2} r_{o} f_{o}^{L \omega} e^{i \omega t}+c e^{i \omega t}}{r_{o}}+\hat{C}_{r z}^{d, L} \varepsilon_{o} e^{i \omega t}
\end{aligned}
$$

Which can be simplified to,

$$
\begin{aligned}
& {\left[\hat{A}_{r}^{L}\left(\Gamma(\bar{\omega})-\Upsilon^{L} \frac{\hat{A}_{z}^{L}}{\hat{A}_{r}^{L}} \varepsilon_{o}-\Upsilon^{L} f_{o}^{L \omega}\right)+f_{o}^{L \omega} \hat{C}_{r r}^{d, L}\right]+\left(\hat{C}_{r \theta}^{d, L}-\hat{C}_{r r}^{d, L}\right)} \\
& {\left[\frac{\hat{A}_{r}^{L}}{\hat{C}_{r r}^{d, L}}\left(\left\{\Theta(\bar{\omega})-\Upsilon^{L} \frac{\hat{A}_{z}^{L}}{2 \hat{A}_{r}^{L}} \varepsilon_{o}-\frac{1}{2} \Upsilon^{L} f_{o}^{L \omega}\right\}\right)+\frac{1}{2} f_{o}^{L \omega}+\frac{c}{r_{o}}\right]+\hat{C}_{r z}^{d, L} \varepsilon_{o}=0}
\end{aligned}
$$


Rewrite Eq. (28a)

$$
\begin{aligned}
& \hat{C}_{r r}^{d, L} f_{o}^{L \omega}+\left(\hat{C}_{r \theta}^{d, L}-\hat{C}_{r r}^{d, L}\right) \\
& {\left[\frac{\hat{A}_{r}^{L}}{a \hat{C}_{r r}^{d, L}}\left(\Theta(\bar{\omega})-\Upsilon^{L} \frac{\hat{A}_{z}^{L}}{2 \hat{A}_{r}^{L}} \varepsilon_{o} a-\Upsilon^{L} f_{o}^{L \omega} \frac{a}{2}\right)+\frac{1}{2} f_{o}^{L \omega}+\frac{c}{r_{o} a^{2}}\right]+\hat{C}_{r z}^{d, L} \varepsilon_{o}=0}
\end{aligned}
$$

Multiply Eq. (32) by $a^{2}$

$$
\begin{aligned}
& a^{2} \hat{C}_{r r}^{d, L} f_{o}^{L \omega}+\left(\hat{C}_{r \theta}^{d, L}-\hat{C}_{r r}^{d, L}\right) \\
& {\left[\frac{a \hat{A}_{r}^{L}}{\hat{C}_{r r}^{d, L}}\left(\Theta(\bar{\omega})-\Upsilon^{L} \frac{\hat{A}_{z}^{L}}{2 \hat{A}_{r}^{L}} \varepsilon_{o} a-\Upsilon^{L} f_{o}^{L \omega} \frac{a}{2}\right)+\frac{a^{2}}{2} f_{o}^{L \omega}+\frac{c}{r_{o}}\right]+a^{2} \hat{C}_{r z}^{d, L} \varepsilon_{o}=0}
\end{aligned}
$$

Now subtract Eq. (33) from Eq. (31a)

$$
\begin{aligned}
& \hat{A}_{r}^{L}\left(\Gamma(\bar{\omega})-\Upsilon^{L} \frac{\hat{A}_{z}^{L}}{\hat{A}_{r}^{L}} \varepsilon_{o}\right)-\hat{A}_{r}^{L} \Upsilon^{L} f_{o}^{L \omega}+f_{o}^{L \omega} \hat{C}_{r r}^{d, L}+ \\
& \left(\hat{C}_{r \theta}^{d, L}-\hat{C}_{r r}^{d, L}\right)\left[\frac{\hat{A}_{r}^{L}}{\hat{C}_{r r}^{d, L}}\left(\left\{\Theta(\bar{\omega})-\Upsilon^{L} \frac{\hat{A}_{z}^{L}}{2 \hat{A}_{r}^{L}} \varepsilon_{o}\right\}\right)-\frac{\hat{A}_{r}^{L}}{2 \hat{C}_{r r}^{d, L}} \Upsilon^{L} f_{o}^{L \omega}+\frac{1}{2} f_{o}^{L \omega}+\frac{c}{r_{o}}\right]+ \\
& \hat{C}_{r z}^{d, L} \varepsilon_{o}-a^{2} \hat{C}_{r r}^{d, L} f_{o}^{L \omega}-\left(\hat{C}_{r \theta}^{d, L}-\hat{C}_{r r}^{d, L}\right) \\
& {\left[\frac{a \hat{A}_{r}^{L}}{\hat{C}_{r r}^{d, L}}\left(\Theta(\bar{\omega})-\Upsilon^{L} \frac{\hat{A}_{z}^{L}}{2 \hat{A}_{r}^{L}} \varepsilon_{o} a\right)-\frac{\hat{A}_{r}^{L}}{2 \hat{C}_{r r}^{d, L}} a^{2} \Upsilon^{L} f_{o}^{L \omega}+\frac{a^{2}}{2} f_{o}^{L \omega}+\frac{c}{r_{o}}\right]-a^{2} \hat{C}_{r z}^{d, L} \varepsilon_{o}}
\end{aligned}
$$

Which can be reduced. Arrangements of terms after that will lead to,

$$
\begin{aligned}
& f_{o}^{L \omega}\left\{\begin{array}{l}
\hat{A}_{r}^{L} \Upsilon^{L}-\hat{C}_{r r}^{d, L}\left(1-a^{2}\right)+ \\
+\left(\hat{C}_{r \theta}^{d, L}-\hat{C}_{r r}^{d, L}\right) \frac{\hat{A}_{r}^{L}}{2 \hat{C}_{r r}^{d, L}} \Upsilon^{L}\left[1-a^{2}\right]-\frac{1}{2}\left(\hat{C}_{r \theta}^{d, L}-\hat{C}_{r r}^{d, L}\right)\left[1-a^{2}\right]
\end{array}\right\} \\
& =\hat{A}_{r}^{L}\left(\Gamma(\bar{\omega})-\Upsilon^{L} \frac{\hat{A}_{z}^{L}}{\hat{A}_{r}^{L}} \varepsilon_{o}\right)+\frac{\hat{A}_{r}^{L}}{\hat{C}_{r r}^{d, L}}\left(\hat{C}_{r \theta}^{d, L}-\hat{C}_{r r}^{d, L}\right)\left(\Theta(\bar{\omega})[1-a]-\Upsilon^{L} \frac{\hat{A}_{z}^{L}}{2 \hat{A}_{r}^{L}} \varepsilon_{o}\left[1-a^{2}\right]\right) \\
& +\hat{C}_{r z}^{d, L} \varepsilon_{o}\left[1-a^{2}\right]
\end{aligned}
$$

Solve for $f_{o}^{L \omega}$ 


$$
\begin{aligned}
& f_{o}^{L \omega}= \\
& \frac{\hat{A}_{r}^{L}\left(\Gamma(\bar{\omega})-\Upsilon^{L} \frac{\hat{A}_{z}^{L}}{\hat{A}_{r}^{L}} \varepsilon_{o}\right)+\frac{\hat{A}_{r}^{L}}{\hat{C}_{r r}^{d, L}}\left(\hat{C}_{r \theta}^{d, L}-\hat{C}_{r r}^{d, L}\right)\left(\Theta(\bar{\omega})[1-a]-\Upsilon^{L} \frac{\hat{A}_{z}^{L}}{2 \hat{A}_{r}^{L}} \varepsilon_{o}\left[1-a^{2}\right]\right)+\hat{C}_{r z}^{d, L} \varepsilon_{o}\left[1-a^{2}\right]}{\left\{\hat{A}_{r}^{L} \Upsilon^{L}-\hat{C}_{r r}^{d, L}\left(1-a^{2}\right)+\left(\hat{C}_{r \theta}^{d, L}-\hat{C}_{r r}^{d, L}\right) \frac{\hat{A}_{r}^{L}}{2 \hat{C}_{r r}^{d, L}} \Upsilon^{L}\left[1-a^{2}\right]-\frac{1}{2}\left(\hat{C}_{r \theta}^{d, L}-\hat{C}_{r r}^{d, L}\right)\left[1-a^{2}\right]\right\}}
\end{aligned}
$$

Eq. (34) can be substituted into Eq. (24a) to obtain an expression for the pore pressure distribution in the osteon.

We can simplify Eq. (34) to become

$$
\begin{aligned}
& \Phi f_{o}^{L \omega}=\hat{A}_{r}^{L}\left(\Gamma(\bar{\omega})-\Upsilon^{L} \frac{\hat{A}_{z}^{L}}{\hat{A}_{r}^{L}} \varepsilon_{o}\right)+ \\
& \frac{\hat{A}_{r}^{L}}{\hat{C}_{r r}^{d, L}}\left(\hat{C}_{r \theta}^{d, L}-\hat{C}_{r r}^{d, L}\right)\left(\Theta(\bar{\omega})[1-a]-\Upsilon^{L} \frac{\hat{A}_{z}^{L}}{2 \hat{A}_{r}^{L}} \varepsilon_{o}\left[1-a^{2}\right]\right)+\hat{C}_{r z}^{d, L} \varepsilon_{o}\left[1-a^{2}\right]
\end{aligned}
$$

Where

$$
\Phi=\left\{\hat{A}_{r}^{L} \Upsilon^{L}-\hat{C}_{r r}^{d, L}\left(1-a^{2}\right)+\left(\hat{C}_{r \theta}^{d, L}-\hat{C}_{r r}^{d, L}\right) \frac{\hat{A}_{r}^{L}}{2 \hat{C}_{r r}^{d, L}} \Upsilon^{L}\left[1-a^{2}\right]-\frac{1}{2}\left(\hat{C}_{r \theta}^{d, L}-\hat{C}_{r r}^{d, L}\right)\left[1-a^{2}\right]\right\}
$$

Recall from Eq. (27)

$$
A_{2}^{\omega}=\frac{\Upsilon^{L}\left[\frac{\hat{A}_{z}^{L}}{\hat{A}_{r}^{L}} \varepsilon_{o}+f_{o}^{L \omega}\right] \mathrm{X}_{1}(\bar{\omega}: \eta)-1-\eta I_{o}(\sqrt{i \bar{\omega}} a)}{\mathrm{X}_{2}(\bar{\omega}: \eta) I_{o}(\sqrt{i \bar{\omega}} a)+K_{o}(\sqrt{i \bar{\omega}} a) \mathrm{X}_{1}(\bar{\omega}: \eta)}
$$

Which can be rewritten as,

$$
A_{2}^{\omega}=\frac{\Sigma(\bar{\omega}: \eta) \Upsilon^{L} f_{o}^{L \omega}}{\mathrm{X}(\bar{\omega}: \eta)}+\frac{\frac{\hat{A}_{z}^{L}}{\hat{A}_{r}^{L}} \varepsilon_{o} \Upsilon^{L} \Sigma(\bar{\omega}: \eta)}{\mathrm{X}(\bar{\omega}: \eta)}
$$

Where 


$$
\begin{gathered}
\mathrm{X}(\bar{\omega}: \eta)=\mathrm{X}_{2}(\bar{\omega}: \eta) I_{o}(\sqrt{i \bar{\omega}} a)+K_{o}(\sqrt{i \bar{\omega}} a) \mathrm{X}_{1}(\bar{\omega}: \eta) \\
\Sigma(\bar{\omega}: \eta)=\Upsilon^{L} \mathrm{X}_{1}(\bar{\omega}: \eta)-1-\eta I_{o}(\sqrt{i \bar{\omega}} a)
\end{gathered}
$$

Recall from Eq.(24),

$$
A_{1}^{\omega}=\frac{\left(\Upsilon^{L}\left[\frac{\hat{A}_{z}^{L}}{\hat{A}_{r}^{L}} \varepsilon_{o}+f_{o}^{L \omega}\right]-A_{2}^{\omega} K_{o}(\sqrt{i \bar{\omega}} a)\right)}{I_{o}(\sqrt{i \bar{\omega}} a)}
$$

Substitute the two constants from Eq. (24) and Eq.(36) into Eq.(31) which will yield,

$$
\Theta(\bar{\omega})=\frac{1}{\sqrt{i \bar{\omega}}}\left\{\frac{\Upsilon^{L}\left[\frac{\hat{A}_{z}^{L}}{\hat{A}_{r}^{L}} \varepsilon_{o}+f_{o}^{L \omega}\right] I_{1}(\sqrt{i \bar{\omega}})}{I_{o}(\sqrt{i \bar{\omega}} a)}-A_{2}^{\omega}\left[\frac{K_{o}(\sqrt{i \bar{\omega}} a) I_{1}(\sqrt{i \bar{\omega}})}{I_{o}(\sqrt{i \bar{\omega}} a)}-K_{1}(\sqrt{i \bar{\omega}})\right]\right\}
$$

To simplify assign,

$$
\mathbb{N}_{1}(\bar{\omega})=\frac{K_{o}(\sqrt{i \bar{\omega}} a) I_{1}(\sqrt{i \bar{\omega}})}{I_{o}(\sqrt{i \bar{\omega}} a)}-K_{1}(\sqrt{i \bar{\omega}})
$$

thus

$$
\Theta(\bar{\omega})=\frac{1}{\sqrt{i \bar{\omega}}}\left\{\frac{\Upsilon^{L}\left[\frac{\hat{A}_{z}^{L}}{\hat{A}_{r}^{L}} \varepsilon_{o}+f_{o}^{L \omega}\right] I_{1}(\sqrt{i \bar{\omega}})}{I_{o}(\sqrt{i \bar{\omega}} a)}-A_{2}^{\omega} \mathbb{N}_{1}(\bar{\omega})\right\}
$$

Recall from Eq. (36)

$$
A_{2}^{\omega}=\frac{\Sigma(\bar{\omega}: \eta) \Upsilon^{L} f_{o}^{L \omega}}{\mathrm{X}(\bar{\omega}: \eta)}+\frac{\frac{\hat{A}_{z}^{L}}{\hat{A}_{r}^{L}} \varepsilon_{o} \Upsilon^{L} \Sigma(\bar{\omega}: \eta)}{\mathrm{X}(\bar{\omega}: \eta)}
$$

Substitute that into the equation of $\Theta(\bar{\omega})$ yield,

$$
\Theta(\bar{\omega})=\frac{1}{\sqrt{i \bar{\omega}}}\left\{\Upsilon^{L} f_{o}^{L \omega}\left[\frac{I_{1}(\sqrt{i \bar{\omega}})}{I_{o}(\sqrt{i \bar{\omega}} a)}-\frac{\Sigma(\bar{\omega}: \eta) \mathbb{N}(\bar{\omega})}{\mathrm{X}(\bar{\omega}: \eta)}\right]+\frac{\hat{A}_{z}^{L}}{\hat{A}_{r}^{L}} \varepsilon_{o} \Upsilon^{L}\left[\frac{I_{1}(\sqrt{i \bar{\omega}})}{I_{o}(\sqrt{i \bar{\omega}} a)}-\frac{\mathbb{N}_{1}(\bar{\omega}) \Sigma(\bar{\omega}: \eta)}{\mathrm{X}(\bar{\omega}: \eta)}\right]\right\}
$$


To simplify assume

$$
\mathbb{Q}_{1}(\bar{\omega}: \eta)=\frac{1}{\sqrt{i \bar{\omega}}}\left[\frac{I_{1}(\sqrt{i \bar{\omega}})}{I_{o}(\sqrt{i \bar{\omega}} a)}-\frac{\Sigma(\bar{\omega}: \eta) \mathbb{N}_{1}(\bar{\omega})}{\mathrm{X}(\bar{\omega}: \eta)}\right]
$$

Thus

$$
\Theta(\bar{\omega})=\Upsilon^{L} f_{o}^{L \omega} \mathbb{Q}_{1}(\bar{\omega}: \eta)+\frac{\hat{A}_{z}^{L}}{\hat{A}_{r}^{L}} \varepsilon_{o} \Upsilon^{L} \mathbb{Q}_{1}(\bar{\omega}: \eta)
$$

Eq. (30) will be expanded,

$$
\begin{gathered}
\Gamma(\bar{\omega})=A_{1}^{\omega} I_{o}(\sqrt{i \bar{\omega}})+A_{2}^{\omega} K_{o}(\sqrt{i \bar{\omega}}) \\
\Gamma(\bar{\omega})=\left[\frac{\left(\Upsilon^{L}\left[\frac{\hat{A}_{z}^{L}}{\hat{A}_{r}^{L}} \varepsilon_{o}+f_{o}^{L \omega}\right]-A_{2}^{\omega} K_{o}(\sqrt{i \bar{\omega}} a)\right]}{I_{o}(\sqrt{i \bar{\omega}} a)}\right] I_{o}(\sqrt{i \bar{\omega}})+A_{2}^{\omega} K_{o}(\sqrt{i \bar{\omega}}) \\
\Gamma(\bar{\omega})=\left\{\frac{\Upsilon^{L}\left[\frac{\hat{A}_{z}^{L}}{\hat{A}_{r}^{L}} \varepsilon_{o}+f_{o}^{L \omega}\right]}{I_{o}(\sqrt{i \bar{\omega}} a)} I_{o}(\sqrt{i \bar{\omega}})+A_{2}^{\omega} K_{o}(\sqrt{i \bar{\omega}})-A_{2}^{\omega} \frac{K_{o}(\sqrt{i \bar{\omega}} a) I_{o}(\sqrt{i \bar{\omega}})}{I_{o}(\sqrt{i \bar{\omega}} a)}\right\} \\
\Gamma(\bar{\omega})=\frac{\Upsilon^{L}\left[\frac{\hat{A}_{z}^{L}}{\hat{A}_{r}^{L}} \varepsilon_{o}+f_{o}^{L \omega}\right]}{I_{o}(\sqrt{i \bar{\omega}} a)} I_{o}(\sqrt{i \bar{\omega}})+A_{2}^{\omega} \mathbb{N}_{2}(\bar{\omega})
\end{gathered}
$$

Where

$$
\mathrm{N}_{2}(\bar{\omega})=K_{o}(\sqrt{i \bar{\omega}})-\frac{K_{o}(\sqrt{i \bar{\omega}} a) I_{o}(\sqrt{i \bar{\omega}})}{I_{o}(\sqrt{i \bar{\omega}} a)}
$$

Thus 


$$
\begin{aligned}
& \Gamma(\bar{\omega})=\frac{\Upsilon^{L}\left[\frac{\hat{A}_{z}^{L}}{\hat{A}_{r}^{L}} \varepsilon_{o}+f_{o}^{L \omega}\right]}{I_{o}(\sqrt{i \bar{\omega}} a)} I_{o}(\sqrt{i \bar{\omega}})+\left[\frac{\Sigma(\bar{\omega}: \eta) \Upsilon^{L} f_{o}^{L \omega}}{\mathrm{X}(\bar{\omega}: \eta)}+\frac{\frac{\hat{A}_{z}^{L}}{\hat{A}_{r}^{L}} \varepsilon_{o} \Upsilon^{L} \Sigma(\bar{\omega}: \eta)}{\mathrm{X}(\bar{\omega}: \eta)}\right] \mathbb{N}_{2}(\bar{\omega}) \\
& \Gamma(\bar{\omega})=\Upsilon^{L} f_{o}^{L \omega}\left[\frac{I_{o}(\sqrt{i \bar{\omega}})}{I_{o}(\sqrt{i \bar{\omega}} a)}+\frac{\Sigma(\bar{\omega}: \eta) \mathbb{N}_{2}(\bar{\omega})}{\mathrm{X}(\bar{\omega}: \eta)}\right]+\frac{\hat{A}_{z}^{L}}{\hat{A}_{r}^{L}} \varepsilon_{o} \Upsilon^{L}\left[\frac{I_{o}(\sqrt{i \bar{\omega}})}{I_{o}(\sqrt{i \bar{\omega}} a)}+\frac{\Sigma(\bar{\omega}: \eta) \mathbb{N}_{2}(\bar{\omega})}{\mathrm{X}(\bar{\omega}: \eta)}\right]
\end{aligned}
$$

assume

$$
\mathbb{Q}_{2}(\bar{\omega}: \eta)=\left[\frac{I_{o}(\sqrt{i \bar{\omega}})}{I_{o}(\sqrt{i \bar{\omega}} a)}+\frac{\Sigma(\bar{\omega}: \eta) \mathbb{N}_{2}(\bar{\omega})}{\mathrm{X}(\bar{\omega}: \eta)}\right]
$$

Thus

$$
\Gamma(\bar{\omega})=\Upsilon^{L} f_{o}^{L \omega} \mathbb{Q}_{2}(\bar{\omega}: \eta)+\frac{\hat{A}_{z}^{L}}{\hat{A}_{r}^{L}} \varepsilon_{o} \Upsilon^{L} \mathbb{Q}_{2}(\bar{\omega}: \eta)
$$

Now expand Eq. (34a)

$$
\begin{aligned}
& \Phi f_{o}^{L \omega}=\hat{A}_{r}^{L} \Gamma(\bar{\omega})-\Upsilon^{L} \hat{A}_{z}^{L} \varepsilon_{o}+\frac{\hat{A}_{r}^{L}}{\hat{C}_{r r}^{d, L}}\left(\hat{C}_{r \theta}^{d, L}-\hat{C}_{r r}^{d, L}\right)[1-a] \Theta(\bar{\omega})- \\
& \left(\hat{C}_{r \theta}^{d, L}-\hat{C}_{r r}^{d, L}\right) \Upsilon^{L} \frac{\hat{A}_{z}^{L}}{2 \hat{C}_{r r}^{d, L}} \varepsilon_{o}\left[1-a^{2}\right]+\hat{C}_{r z}^{d, L} \varepsilon_{o}\left[1-a^{2}\right]
\end{aligned}
$$

Successive algebraic operations and substitutions will lead to

$$
\begin{aligned}
& f_{o}^{L \omega}= \\
& \frac{\left\{\Phi-\hat{A}_{r}^{L} \Upsilon^{L} \mathbb{Q}_{2}(\bar{\omega}: \eta)-\frac{\hat{A}_{r}^{L}}{\hat{C}_{r r}^{d, L}}\left(\hat{C}_{r \theta}^{d, L}-\hat{C}_{r r}^{d, L}\right)[1-a] \Upsilon^{L} \mathbb{Q}_{1}(\bar{\omega}: \eta)\right\}}{\varepsilon_{o} \hat{A}_{z}^{L} \Upsilon^{L} \mathbb{Q}_{2}(\bar{\omega}: \eta)+\frac{\hat{A}_{z}^{L}}{\hat{C}_{r r}^{d, L}}\left(\hat{C}_{r \theta}^{d, L}-\hat{C}_{r r}^{d, L}\right)[1-a] \varepsilon_{o} \Upsilon^{L}\left(\mathbb{Q}_{1}(\bar{\omega}: \eta)-\frac{[1+a]}{2}\right)+\hat{C}_{r z}^{d, L} \varepsilon_{o}\left[1-a^{2}\right]}
\end{aligned}
$$

\section{Results}

Equation (40) above defines the last constant that need to be determined. Now Tables (1) and (2) above can be used to define the pore pressure distribution in time domain and location.

$$
p^{L}(\lambda, t)=\left\{A_{1}^{\omega} I_{o}(\sqrt{i \bar{\omega}} \lambda)+A_{2}^{\omega} K_{o}(\sqrt{i \bar{\omega}} \lambda)-\Upsilon^{L} \frac{\hat{A}_{z}^{L}}{\hat{A}_{r}^{L}} \varepsilon_{o}-\Upsilon^{L} f_{o}^{L \omega}\right\} e^{i \omega t}
$$

The relationship between the pore pressure and the dimensionless radius for different values of leakage parameter is shown in Fig. 2 below. The results for leakage paramour of values of 1 and 0 
are consistent with the results demonstrated in the literature) for no flow from the outer boundaries and free flow from the outer boundaries ${ }^{16}$. To get in depth understanding of the relationship between the leakage and the pore pressure, Fig. 3 is plotted based on the results of Fig. 2. The figure shows that the relationship between the pore pressure in the cement line and the corresponding leakage is quasi linear or not perfectly linear.

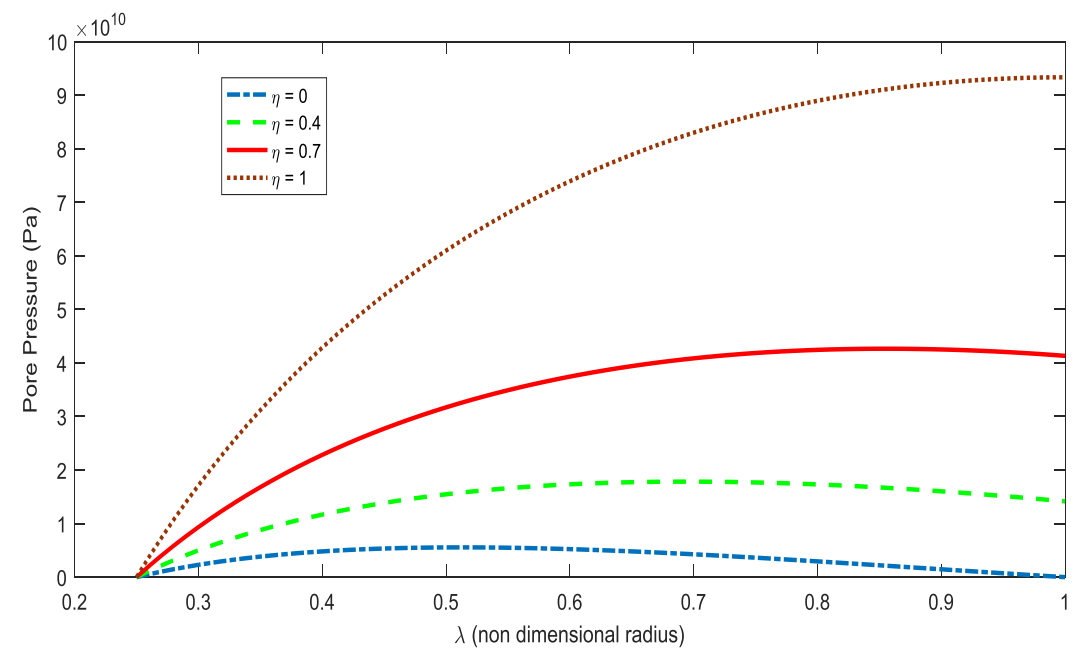

Fig. 2: Variation of the pore pressure with the non dimensional radius of the osteon for different values of the leakage.

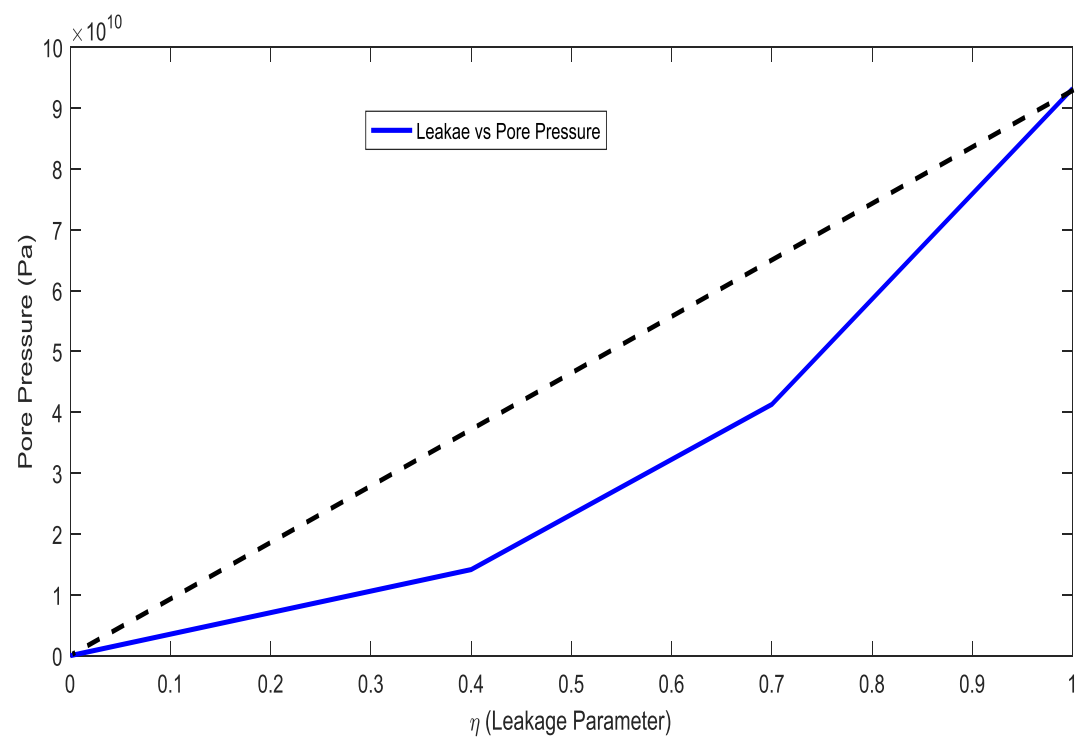

Fig. 2: Variation of the pore pressure on the cement line with the leakage parameter

\section{Conclusion}


There are very few attempts to quantify theoretically the flow through the cement line. The method presented targeted specific type of load for specific conditions to determine the effect of leakage through the cement line. More experimental and theoretical work needs to be done to identify the sensitivity of the mechanical properties of the osteon due to change in leakage. Experimental work will be very challenging due to the small size of the cement line (less than10 $\mathrm{n} m$ in wall thickness).

\section{Acknowledgment}

The authors acknowledge the funding from CUNY-CIRG and NSF-ATE 1601522

\section{References}

1. Ascenzi M, Benvenuti A, Mango F, Similia R, Mechanical hystersis loops from single osteons: Technical Devices and Preliminary Results, ASME J. of Biomech, 18(5), pp. 391-398, 1985.

2. Ascenzi A, Baschieri P, Benvenuti A, The Torsional Properties of Single Selected Osteons, ASME J. of Biomech, 27(7), pp. 875-884, 1994.

3. Frasca P, Harper R. A, Katz J. L, Isolation of Single Osteons and Osteon Lamellae, Acta Anat 95:122129, 1976.

4. Gailani G, Benalla M, Mahamud R, Cowin S, Cardoso L, Experimental determination of the permeability of the lacunar canalicular porosity of bone, J. of Biomechanical Engineering, v131-10, pp101007 (7pages), 2009.

5. Schaffler M, Burr D, Frederickson R, Morphology of the osteonal cement line, Anat Rec. 1987 Mar, 217(3), 223-8

6. Cowin S. C, Bone mechanics hand book, CRC Press, ISBN 0-8493-9117-2, pp1 - 10, 2001.

7. Lakes R, Saha S, Cement line motion in bone, Science, Volume 204, Issue 4392, pp. 501-503, 1979.

8. Park H, Lakes R, Cosserat micromechanics of human bone: strain redistribution by a hydration-sensitive constituent, J. Biomechanics, 19 385-397, 1986

9. Rémond A, Naili S, Transverse isotropic poroelastic osteon model under cyclic Loading, Mech. Res, Comm. 32: 645-651, 2005.

10. Dong X, Zhang X, Guo X, Interfacial Strength of Cement Lines in Human Cortical Bone, MCB, 2(2), 63 $-68,2005$.

11. Wang L, Fritton SP, Cowin S, Weinbaum S, Fluid pressure relaxation mechanisms in osteonal bone specimens: modeling of an oscillatory bending experiment, J. of Biomech 32: 663-72, 1999.

12. Armstrong $\mathrm{C}$, Lai $\mathrm{W}$, Mow $\mathrm{V}$, An analysis of the unconfined compression of articular cartilage, J. of Biomech Engr, 106:165-173, 1984.

13. Cheng S, Bilston L, Unconfined compression of white matter, J. of Biomech, 40, 117-124, 2007.

14. Cohen B, Lai W, Mow V, A transversely isotropic biphasic model for unconfined compression of growth plate and chondroepiphysis, J. of Biomech Engr. 120: 491-6, 1998.

15. Yin L, Elliott DM, A biphasic and transversely isotropic mechanical model for tendon: application to mouse tail fascicles in uniaxial tension, J. of Biomech. 37: 907-16.

16. Gailani G, Cowin S, The unconfined compression of a poroelastic annular cylindrical disk, J. of Mech. of Materials, 40(6), $507-23$.

17. Cowin S, Mehrabadi M, Compressible and incompressible constituents in anisotropic poroelasticity: the problem of unconfined compression of a disk, J. Mech. Phys. Solids 55, 161-193, 2007.

18. Cowin S, Gailani G, Benalla M, Hierarchical poroelasticity: movement of interstitial fluid between porosity levels in bones, Philos. Trans. R. Soc. A Mech. Biol. 367, 3401-3444, 2009. 
